\title{
NONLINEAR EIGENVALUE PROBLEMS AND GALERKIN APPROXIMATIONS
}

\author{
BY FELIX E. BROWDER
}

Communicated December 21, 1967

Let $X$ be a reflexive Banach space, $T$ and $S$ two mappings of $X$ into its conjugate space $X^{*}$. We denote the pairing between $w$ in $X^{*}$ and $u$ in $X$ by $(w, u)$, and weak convergence (in either $X$ or $X^{*}$ ) by $\rightarrow$, strong convergence (in either $X$ or $X^{*}$ ) by $\rightarrow$.

By an eigenvalue problem for the pair $(T, S)$, we mean the problem of finding an element $u$ in $X$ and a real number $\lambda$ such that

$$
T(u)=\lambda S(u),
$$

with $u$ possibly satisfying additional normalization conditions. It is our purpose in the present note to describe a way of applying a method of Galerkin type to such problems which works in particular for nonlinear elliptic boundary value problems of variational type. We obtain from it a general theorem on the existence of normalized eigenfunctions for the latter problem, and in the case of $T$ and $S$ odd operators, we obtain also an extremely general form of a theory of Lusternik-Schnirelman type guaranteeing the existence of infinitely many distinct normalized eigenfunctions.

We consider first some restrictions that may be placed on the nonlinear operator $T$.

DEFINITION 1. $T$ is said to satisfy condition $(S)$ if for any sequence $\left\{u_{j}\right\}$ in $X$ with $u_{j} \rightarrow u$ in $X$ and $\left(T\left(u_{j}\right)-T(u), u_{j}-u\right) \rightarrow 0$, we have $u_{j} \rightarrow u$ in $X$.

DEFINITION 2. $T$ is said to satisfy condition $(S)_{0}$ if for each sequence $\left\{u_{j}\right\}$ in $X$ with $u_{j} \rightarrow u$ in $X, T\left(u_{j}\right) \rightarrow z$ in $X^{*}$, and $\left(T\left(u_{j}\right), u_{j}\right) \rightarrow(z, u)$, we have $u_{j} \rightarrow u$ in $X$.

Lemma 1. (a) If T satisfies condition (S), it satisfies condition $(S)_{0}$.

(b) If $T$ is continuous and satisfies condition $(S)_{0}$, and if $K$ is any compact set of $X^{*}, B$ any bounded closed set of $X$, then $T^{-1}(K) \cap B$ is compact.

(c) If $T$ is continuous and satisfies condition $(S)_{0}$, then the image under $T$ of any bounded closed set $B$ of $X$ is closed in $X^{*}$.

Proof of Lemma 1. Proof of (a). Suppose $u_{j} \rightarrow u, T\left(u_{j}\right) \rightarrow z$, and $\left(T\left(u_{j}\right), u_{j}\right) \rightarrow(z, u)$. Then 


$$
\begin{array}{r}
\left(T\left(u_{j}\right)-T(u), u_{j}-u\right)=\left(T\left(u_{j}\right), u_{j}\right)-\left(T\left(u_{j}\right), u\right)-\left(T(u), u_{j}-u\right) \\
\rightarrow(z, u)-(z, u)-0=0 .
\end{array}
$$

Hence by the condition $(S), u_{j} \rightarrow u$.

Proof of (b). Let $\left\{u_{j}\right\}$ be a sequence in $T^{-1}(K) \cap B$. By passing to a subsequence, we may assume that $u_{j} \rightarrow u$ in $X, T\left(u_{j}\right) \rightarrow z$ in $K$. Hence $\left(T\left(u_{j}\right), u_{j}\right) \rightarrow(z, u)$ and, by condition $(S)_{0}, u_{j} \rightarrow u$. Hence $u \in B$, and by the continuity of $T, T(u)=z$, i.e. $u \in T^{-1}(K) \cap B$.

Q.E.D.

PROOF OF (c). The conclusion of (b) implies that $T$ is a proper continuous map of $B$ into $X^{*}$. Hence it is a closed map of $B$ into $X^{*}$ and $T(B)$ is closed in $X^{*}$.

Q.E.D.

We now give our principal methodological result.

Theorem 1. Let $X$ be a separable reflexive Banach space, $T$ and $S$ two continuous bounded mappings of $X$ into $X^{*}$ with $T$ satisfying condition $(S)_{0}$ and $S$ a compact map of $X$ into $X^{*}$. Let $\left\{X_{n}\right\}$ be an increasing sequence of finite dimensional subspaces of $X$ whose union is dense in $X$, $B$ a closed bounded subset of $X$. Suppose that for each $n$, there exists an element $u_{n}$ of $B \cap X_{n}$ with the property that

$$
j_{n}^{*} T\left(u_{n}\right)=\lambda_{n} j_{n}{ }^{*} S\left(u_{n}\right),
$$

where $j_{n}$ is the injection mapping of $X_{n}$ into $X$, and $j_{n}^{*}$ is the dual projection of $X^{*}$ onto $X_{n}^{*}$. Suppose that $\left|\lambda_{n}\right|$ is uniformly bounded.

Then there exists an eigenfunction $u$ of the pair $(T, S)$ in $B$, i.e. $T(u)$ $=\lambda S(u)$, and for any weakly convergent subsequence $u_{n(k)} \rightarrow u$ of the sequence $\left\{u_{n}\right\}, u$ is such an eigenfunction and $u_{n(k)} \rightarrow u$.

Proof of Theorem 1 . Since $B$ is bounded and $X$ is reflexive, the sequence $\left\{u_{n}\right\}$ has a weakly convergent subsequence. We may replace the original sequence by this subsequence and assume that $u_{n} \rightarrow u$. It suffices to show that $\left\{u_{n}\right\}$ has a strongly convergent subsequence and that $u$ is an eigenfunction of the pair $(T, S)$. Since $\left|\lambda_{n}\right|$ is uniformly bounded, we may assume for our original sequence (again by passing to an infinite subsequence) that $\lambda_{n} \rightarrow \lambda$, and since $S$ is compact, that $S\left(u_{n}\right) \rightarrow w$ in $X^{*}$.

Let $v$ be any element of $V_{m}$ for some $m$, and consider $n \geqq m$. Then,

$$
\left(T u_{n}, v\right)=\left(T u_{n}, j_{n} v\right)=\left(j_{n}^{*} T\left(u_{n}\right), v\right)=\lambda_{n}\left(j_{n}^{*} S\left(u_{n}\right), v\right)=\lambda_{n}\left(S\left(u_{n}\right), v\right) .
$$

Hence

$$
\left(T\left(u_{n}\right), v\right) \rightarrow \lambda(w, v),(n \rightarrow+\infty) .
$$

Since this is true for each $v$ in the dense union of the spaces $V_{m}$ and since the sequence $\left\{T\left(u_{n}\right)\right\}$ is bounded, it follows that $T\left(u_{n}\right) \rightarrow \lambda w$. 
On the other hand, by the same argument,

$$
\left(T\left(u_{n}\right), u_{n}\right)=\lambda_{n}\left(S\left(u_{n}\right), w\right) \rightarrow \lambda(w, v) .
$$

Applying the condition $(S)_{0}$ for $T$, we see that $u_{n} \rightarrow u$. Since $T$ and $S$ are continuous, $T\left(u_{n}\right) \rightarrow T(u), S\left(u_{n}\right) \rightarrow w$. Hence

$$
T(u)=\lim _{n} T\left(u_{n}\right)=\lambda w=-S(u) .
$$

The special interest of the conditions $(S)$ and $(S)_{0}$ is that they are satisfied by quasi-linear elliptic differential operators in generalized divergence form under extremely weak hypotheses on the operators.

THEOREM 2. Let $\Omega$ be a bounded open set in $R^{n}$ for which the Sobolev Imbedding Theorem is valid, $A$ and $B$ two differential operators on $\Omega$ of the form

$$
\begin{aligned}
& A(u)=\sum_{|\alpha| \leqq m}(-1)^{|\alpha|} D^{\alpha} A_{\alpha}\left(x, u, D u, \cdots, D^{m} u\right), \\
& B(u)=\sum_{|\beta| \leqq m-1}(-1)^{|\beta|} D^{\beta} B_{\beta}\left(x, u, \cdots, D^{m} u\right) .
\end{aligned}
$$

For each $\alpha$ and $\beta$, let $A_{\alpha}(x, \xi)$ and $B_{\beta}(x, \xi)$ be continuous in $x$ and Lebesgue measurable in $\xi$. Suppose that for a given exponent $p$ with $1<p<+\infty, V$ is a closed subspace of the Sobolev space $W^{m, p}(\Omega)$ and for $u$ and $v$ in $V$, we set

$$
\begin{aligned}
& a(u, v)=\sum_{|\alpha| \leq m}\left(A_{\alpha}\left(x, u, D u, \cdots, D^{m} u\right), D^{\alpha} v\right), \\
& b(u, v)=\sum_{|\beta| \leqq m-1}\left(B_{\beta}\left(x, u, D u, \cdots, D^{m} u\right), D^{\beta} v\right),
\end{aligned}
$$

(with $\left.(w, v)=\int_{\Omega} w v\right)$. Suppose that the following three conditions are satisfied:

(1) There exists a constant $c_{0}$ and functions $c_{\alpha}$ in $L^{p^{\prime}}(\Omega)$ such that

$$
\begin{aligned}
& \left|A_{\alpha}(x, \xi)\right| \leqq c_{\alpha}(x)+c_{0} \sum_{|\phi|=m}\left|\xi_{\phi}\right|^{p-1}+\sum_{|\phi| \leqq m-1}\left|\xi_{\phi}\right|^{q_{\alpha}}, \\
& \left|B_{\beta}(x, \xi)\right| \leqq c_{\beta}(x)+c_{0} \sum_{|\phi|=m}\left|\xi_{\phi}\right|^{q_{\beta \phi}},
\end{aligned}
$$

where

$$
\begin{aligned}
q_{\alpha \phi}<p_{\phi} q_{\alpha}^{-1}, \quad q_{\alpha} & =\max \left(1, n p(n p-n+p(m-|\alpha|))^{-1}\right), \\
p_{\phi}^{-1} & =\max \left(0, n p(n-p(m-|\phi|))^{-1}\right) .
\end{aligned}
$$

(2) For $\psi=\left\{\psi_{\beta}:|\beta| \leqq m-1\right\}, \zeta=\left\{\zeta_{\alpha}:|\alpha|=m\right\}$, set $A_{\alpha}(x, \psi, \zeta)$ 
$=A(x, \xi)$ where $\xi=[\psi, \zeta]$. Then for every $x$ in $\Omega, \psi, \zeta$ and $\zeta^{\prime}$ with $\zeta \neq \zeta^{\prime}$,

$$
\sum_{|\alpha|=m}\left[A_{\alpha}(x, \psi, \zeta)-A_{\alpha}\left(x, \psi, \zeta^{\prime}\right)\right]\left(\zeta_{\alpha}-\zeta_{\alpha}^{\prime}\right)>0 .
$$

(3) There exist positive constants $c_{1}$ and $c_{2}$ such that

$$
\sum_{|\alpha| \leq m} A_{\alpha}(x, \xi) \xi_{\alpha} \geqq c_{1}|\xi|^{p}-c_{2} .
$$

Then: (a) The form $a(u, v)$ is well defined for all $u$ and $v$ in $V$ and there exists an unique element $T(u)$ in $V^{*}$ such that $a(u, v)=(T(u)$, v) for all $v$ in $V$ and $a$ given element $u$ in $V$. Similarly, $b(u, v)$ is well defined for $u$ and $v$ and $b(u, v)=(S(u), v)$ for all $v$ in $V$ and a given $u$ in $V$, when $S(u) \in V^{*}$.

(b) $T$ is a bounded continuous mapping of $V$ into $V^{*}$ which satisfies condition (S).

(c) $S$ is a compact mapping of $V$ into $V^{*}$.

The proof of Theorem 2 and the details of further applications of these arguments will be given in another paper.

Let us consider, however, the application of Theorems 1 and 2 to the "self-adjoint" case, i.e. when $A$ and $B$ are the Euler-Lagrange operators of multiple integral variational problems.

TheOREM 3. Let $T$ and $S$ be the derivatives of two $C_{1}$ functions $f$ and $g$ on $V$, respectively, where $T$ is bounded and satisfies condition $(S)_{0}$ and $S$ is compact. Let $c$ be a constant such that on the level set $M_{c}$ $=\{u \mid f(u)=c\},(T(u), u)>0$, while $M_{c}$ is bounded. Suppose that $g(u)>0$ for $u$ in $M_{c}$, that $(S(u), u)>0$ on $M_{c}$, and that for each set $B$ on $M_{c}$ for which $g(u)>\epsilon>0,(S(u), u)>d(\epsilon)>0$.

Then $g$ assumes its maximum at a point $u_{0}$ of $M_{c}$, and $T\left(u_{0}\right)=\lambda S\left(u_{0}\right)$ for some $\lambda>0$.

Proof of Theorem 3. $V$ is assumed as in Theorem 1 to be a separable reflexive Banach space. We choose an increasing sequence $V_{n}$ of finite dimensional subspaces whose union is dense in $V$ and with $M_{c} \cap V_{n}$ having their union dense in $M_{c}$. Let $f_{n}$ and $g_{n}$ be the restrictions of $f$ and $g$ to $V_{n}$. Then $M_{c} \cap V_{n}$ is the $c$-level set of $f_{n}$ and $f_{n}^{\prime}$ $=j_{n}{ }^{*} T, g_{n}{ }^{\prime}=j_{n}{ }^{*} S$. Since $\left(f_{n}^{\prime}(u), u\right)=(T(u), u)>0$ on $M_{c} \cap V_{n}, M_{c} \cap V_{n}$ is a manifold. The function $g$ is $C^{1}$ on this compact manifold and assumes its maximum $m_{n}$ on $M_{c} \cap V_{n}$ at a point $u_{n}$ which satisfies the condition $T\left(u_{n}\right)=\lambda_{n} S\left(u_{n}\right)$. Since $g\left(u_{n}\right)=m_{n} \rightarrow m=\sup _{u \in M_{c}} g(u)$, $\left(S\left(u_{n}\right), u_{n}\right) \geqq d_{0}>0$ for all $n$. Hence, since

$$
\lambda_{n}=\left(T\left(u_{n}\right), u_{n}\right) /\left(S\left(u_{n}\right), u_{n}\right),
$$


$\lambda_{n}$ is uniformly bounded. If we apply Theorem 1 , we obtain the conclusion that for an infinite subsequence, $u_{n(k)} \rightarrow u$, where $u$ is an eigenfunction $T(u)=\lambda S(u)$. Since $g$ is continuous, $g\left(u_{n(k)}\right) \rightarrow g(u)=m$. Since $M_{c}$ is closed, $u \in M_{c}$.

Q.E.D.

Theorem 4. Let $V$ be a separable reflexive Banach space, $T$ and $S$ two continuous mappings of $V$ into $V^{*}$ with $T$ bounded and satisfying condition $(S)_{0}, S$ compact. Suppose that $T$ and $S$ are the derivatives of two $C^{1}$ functions $f$ and $g$ on $V$, and suppose that on the level set $M_{c}$ $=\{u \mid f(u)=c\},(T(u), u)>0$. Suppose that $M_{c}$ is invariant under the involution $\pi(u)=-u$, and that $g(-u)=g(u)$ on $M_{c}$. Suppose further that $M_{c}$ is intersected exactly once by each ray through the origin, that $g(u)>0$ for $u$ in $M_{c}$, that $(S(u), u)>0$ on $M_{c}$ and that $g(u)$ and $(S(u), u)$ go to zero together on $M_{c}$. Suppose finally that for each $\epsilon>0$, there exists a finite dimensional subspace $V_{\mathrm{\epsilon}}$ of $V$ such that outside the $\epsilon$-neighborhood of $V_{\epsilon}, g(u)<\epsilon$. For each $j$, let

$$
h_{j}=\sup _{p-\operatorname{cat}\left(K, M_{0}\right) \geq j} \min _{u \in K} g(u),
$$

where the supremum is taken over compact subsets $K$ of $M_{c}$ whose image in $M_{c} / \pi$ has Lusternik-Schnirelman category $\geqq j$.

Then:

(a) For each $j, h_{j}$ is well defined and there exists $u_{j}$ in $M_{c}$ with

$$
T\left(u_{j}\right)=\lambda_{j} S\left(u_{j}\right),\left(\lambda_{j}>0\right), \quad f\left(u_{j}\right)=c, \quad g\left(u_{j}\right)=h_{j},
$$

while $\lambda_{j} \rightarrow+\infty, h_{j} \rightarrow 0$.

(b) Suppose that $\operatorname{dim}\left(V_{n}\right) \geqq j$. Then we can define

$$
h_{j, n}=\sup _{p-\operatorname{cat}\left(K, M_{\mathrm{o}}\right) \geq j, K \subset V_{n}} \min _{u \in K} g(u),
$$

and for each $j \leqq n$, there exists $u_{j, n}$ in $V_{n}$ such that

$$
j_{n}^{*} T\left(u_{j, n}\right)=j_{n}^{*} S\left(u_{j, n}\right), \quad f\left(u_{j, n}\right)=c, \quad g\left(u_{j, n}\right)=h_{j, n} .
$$

(c) For any fixed $j$ and any infinite subsequence $u_{j, n(k)} \rightarrow u_{j}$ as $k \rightarrow \infty$, $u_{j}$ is an eigenfunction satifsying the condition of part (a) and $u_{j, n(k)} \rightarrow u_{j}$.

Proof of Theorem 4. Since $f_{n}^{\prime}=j_{n}^{*} T$, so that $\left(f_{n}^{\prime}(u), u\right)>0$ on $M_{c} \cap V_{n}$, the latter is a manifold for each $n$, and $\left(M_{c} \cap V_{n}\right) / \pi$ is homeomorphic to $P^{n-1}$, which has Lusternik-Schnirelman category $n$. The conclusions of (b) then follow from the classical LusternikSchnirelman theory on finite dimensional manifolds (Lusternik [7], Vainberg [8]). The conclusion of (a) will follow from that of part (c) so that it suffices to prove (c). 
Proof of (c). We may assume without loss of generality that $u_{j, n} \rightarrow u_{j}$ as $n \rightarrow \infty$. Since $g\left(u_{j, n}\right)=h_{j, n} \rightarrow h_{j}$ as $j \rightarrow+\infty$ where $h_{j}>0$ for each $j$, it follows that $\left(S\left(u_{j, n}\right), u_{j, n}\right) \geqq d_{0}>0$ for all $n$. Hence $\lambda_{j, n}$ $=\left(T\left(u_{j, n}\right), u_{j, n}\right)\left(S\left(u_{j, n}\right), u_{j, n}\right)^{-1}$ is uniformly bounded. Applying Theorem 1, we find that $u_{j, n} \rightarrow u_{j}$. Hence $f\left(u_{j}\right)=\lim _{n} f\left(u_{j, n}\right)=c$. Since $g\left(u_{j}\right)=\lim _{n} g\left(u_{j, n}\right)=h_{j}$, and since by Theorem $1, u_{j}$ is an eigenfunction of the pair $(T, S)$, our conclusion follows.

Q.E.D.

Remarks. (1) The result of Theorem 4 combined with Theorem 2 generalizes the writer's results in [4] under weaker regularity and boundedness hypotheses on the $A_{\alpha}$ and makes no explicit use of the theory of infinite dimensional manifolds.

(2) An earlier attempt to weaken the regularity hypotheses of [4] was made by $M$. Berger [1] using an infinite dimensional argument. His argument in [1] contains a number of serious errors and gaps which make it doubtful that the argument can be carried through (cf. the review by C. W. Clark in Math. Reviews).

(3) A recent paper with a similar title by S. Hildebrandt [6] has no intersection with the present paper since it concerns linear operators depending nonlinearly on $\lambda$, not nonlinear operators depending linearly on $\lambda$. However, the methods of the present paper can be used to combine Hildebrandt's results with those given here and extend them to nonlinear operators.

\section{BIBLIOGRAPHY}

1. M. Berger, A Sturm-Liouville Theorem for nonlinear elliptic partial differential equations, Ann. Scuola Norm. Sup. Pisa 20 (1966), 543-582.

2. F. E. Browder, Nonlinear elliptic boundary value problems, Bull. Amer. Math. Soc. 69 (1963), 862-874.

3. - Variational methods for nonlinear elliptic eigenvalue problems, Bull. Amer. Math. Soc. 71 (1965), 176-183.

4. - Infinite dimensional manifolds and nonlinear elliptic eigenvalue problems, Ann. of Math. 82 (1965), 459-477.

5. - Problèmes nonlinéaires, University of Montreal Press, Montreal, 1966.

6. S. Hildebrandt, Über die Lösung nichtlinearer Eigenwertaufgaben mit der Gälerkinverfahren, Math. Z. 101 (1967), 255-264.

7. L. A. Lusternik, The topology of the calculus of variations in the large, Transl. Math. Monographs, vol. 16, Amer. Math. Soc., Providence, R. I., 1966.

8. M. M. Vainberg, Variational methods for the study of nonlinear operators, Holden-Day, San Francisco, 1964.

University of Chicago 